\title{
Comparative analysis of carboplatin and paclitaxel combination chemotherapy schedules in previously untreated patients with advanced non-small cell lung cancer
}

\author{
TOSHIKI SHIMIZU, TAKASHI YOKOI, TAKESHI TAMAKI, KAYOKO KIBATA, \\ NORIKO INAGAKI and SHOSAKU NOMURA
}

First Department of Internal Medicine, Kansai Medical University, Moriguchi, Osaka 570-8506, Japan

Received October 21, 2012; Accepted January 11, 2013

DOI: 10.3892/ol.2013.1134

\begin{abstract}
The combination of carboplatin and paclitaxel is one of the most commonly used regimens for the treatment of non-small cell lung cancer (NSCLC). We aimed to compare the standard tri-weekly and weekly schedules of this treatment, while considering treatment-related hematological toxicities. We retrospectively analyzed the weekly [paclitaxel, $70 \mathrm{mg} / \mathrm{m}^{2} /$ week on days 1,8 and 15, and carboplatin, area under the curve $(\mathrm{AUC})=6$, every 4 weeks] and standard tri-weekly (paclitaxel, $200 \mathrm{mg} / \mathrm{m}^{2}$, and carboplatin, $\mathrm{AUC}=6$, on day 1 every 3 weeks] schedules in patients with previously untreated advanced NSCLC. A total of 167 patients were enrolled in this study. The median age of the patients was 65 years (range, $31-79$ years). The weekly and standard arms included 73 and 94 patients, respectively. The incidence of grade 3 or 4 neutropenia and neuropathy was significantly decreased in the weekly arm compared with the standard arm (37.0 vs. 70.2\%). The median survival and progression-free survival times were 11.8 and 4.2 months, respectively, in the weekly arm and 11.6 and 3.1 months, respectively, in the standard arm. The results of the multivariate analysis indicated that the weekly schedule [hazard ratio $(\mathrm{HR})=0.634, \mathrm{P}=0.0262$ ] and grade 3 or 4 neutropenia $(\mathrm{HR}=0.372, \mathrm{P}=0.0007)$ were independent favorable prognostic factors for overall survival time. In conclusion, the weekly schedule of carboplatin and paclitaxel was less toxic than and potentially superior to the standard tri-weekly schedule. However, further optimization of the dose and schedule is warranted.
\end{abstract}

\section{Introduction}

Lung cancer is the leading cause of cancer-related mortality worldwide. Platinum-based chemotherapy is recommended as

Correspondence to: Dr Toshiki Shimizu, First Department of Internal Medicine, Kansai Medical University, 10-15 Fumizono-cho, Moriguchi-City, Osaka 570-8506, Japan

E-mail: shimizto@takii.kmu.ac.jp

Key words: non-small cell lung cancer, weekly, carboplatin, paclitaxel, neutropenia a front-line chemotherapy for advanced non-small cell lung cancer (NSCLC) (1). The results of the Four Arms Comparative Study, which analyzed 4 platinum-doublet regimens as frontline therapies for advanced NSCLC, demonstrated that the 4 regimens had similar efficacy $(2,3)$. Thus, carboplatin and paclitaxel combination therapy has been recognized as a reference regimen based on its feasibility. In the standard schedule, $200 \mathrm{mg} / \mathrm{m}^{2}$ paclitaxel and carboplatin [area under the curve $(\mathrm{AUC})=6]$ are administered on day 1 every 3 weeks. However, several weekly schedules have been investigated to reduce hematological toxicities (4-6). Hirabayashi et al also reported a weekly schedule based on the metronomic theory (7). In this weekly schedule, $70 \mathrm{mg} / \mathrm{m}^{2}$ paclitaxel on days 1,8 and 15 , and $\mathrm{AUC}=6$ of carboplatin on day 1 were administered every 4 weeks. All the studies, including the present study, demonstrated that a weekly schedule reduced neutropenia and displayed a comparable survival benefit compared to the standard schedule.

The survival benefit of treatment-related neutropenia has been discussed in previous studies (8-11). Consequently, a treatment schedule correlated with a reduced incidence of neutropenia ought to be an unfavorable predictive factor for overall survival (OS). However, a weekly schedule associated with reduced neutropenia demonstrated a comparable survival benefit to the standard schedule. Notably, there has been no integrated analysis comparing the 2 schedules and concurrently considering the worst grade of treatment-related hematological toxicities. Therefore, we performed a retrospective analysis in unselected patients who received standard tri-weekly or weekly carboplatin in combination with paclitaxel to obtain complimentary information regarding whether the treatment schedule and treatment-related hematological toxicities were correlated with survival outcome.

\section{Patients and methods}

Data collection. The medical records of all patients with NSCLC who were treated between January 1999 and December 2010 at Kansai Medical University Takii Hospital (Moriguchi, Japan) were retrospectively reviewed; institutional review board approval was obtained for this study. The patients were included in this study if they had 
advanced NSCLC (stage IIIB or IV) that was treated with front-line combination chemotherapy including carboplatin and paclitaxel. The patients were assigned to 1 of 2 groups; the weekly arm group (weekly schedule) and the standard arm group (standard tri-weekly schedule). The clinical stage was assigned on the basis of the Sixth Edition of the TNM Classification for Lung Cancer $(12,13)$. Data including gender, age, smoking history, clinical stage, histological cancer type, history of administration of an epidermal growth factor receptor tyrosine kinase inhibitor (EGFR-TKI), Eastern Cooperative Oncology Group (ECOG) performance status (PS), progression-free survival (PFS) and OS were obtained retrospectively from the patient medical records. There was no distinction between gefitinib and erlotinib, and both agents were considered as EGFR-TKIs. Patients who underwent previous palliative radiation treatment, including wholebrain irradiation without curative intention, were included. The crossover cases in the consecutive treatment courses between the weekly and standard arms were excluded, as well as patients with large cell neuroendocine carcinoma. All patients provided informed consent prior to receiving frontline chemotherapy. The study was performed according to the Declaration of Helsinki and was approved by the Institutional Ethics Review Board (the Clinical Research Board of Kansai Medical University Takii Hospital; ID No, 23-6).

Statistical analysis. Differences between the groups were compared using the $\chi^{2}$ or Fisher's exact test. OS was defined as the time from the start of front-line systemic chemotherapy to the time of death from any cause or the date the patient was last known to be alive. PFS was defined as the time between the start of treatment and disease progression, death or the last known follow-up. The treatment-related adverse effects were evaluated using the Common Terminology Criteria for Adverse Events version 4.0 (14). Objective tumor responses to chemotherapy were evaluated using the Response Evaluation Criteria in Solid Tumors version 1.0 (15). The objective response rate (ORR) was defined as the number of patients displaying a complete response (CR) or a partial response (PR), with respect to the total number of patients evaluated. The disease control rate (DCR) was defined as the number of patients displaying a CR, a PR or stable disease (SD), with respect to the total number of patients evaluated. The minimum time interval between the 2 measurements required for the determination of SD was 6 weeks. The 95\% confidence intervals (95\% CIs) for the ORR and DCR were calculated using a binomial distribution. The univariate and multivariate analyses of PFS and OS were performed with the KaplanMeier product-limit method using the log-rank test and the Cox proportional hazards model, respectively. The $95 \%$ CI for the survival rate was calculated using Greenwood's method, and that of the median survival time (MST) was caluclated by the Brookmeyer and Crowley method. All statistical analyses were conducted using JMP (version 9.0.2) software for Windows (SAS Institute Inc, Cary, NC). All statistical tests were two-sided, and $\mathrm{P}<0.05$ was considered to indicate a statistically significant difference.

Treatment plan. In the weekly arm (weekly schedule), $70 \mathrm{mg} / \mathrm{m}^{2}$ paclitaxel was administered on days 1,8 and 15 together with carboplatin $(\mathrm{AUC}=6)$ on day 1 of each 4 -week cycle. In the standard arm (standard tri-weekly schedule), $200 \mathrm{mg} / \mathrm{m}^{2}$ paclitaxel was administered with carboplatin $(\mathrm{AUC}=6)$ on day 1 of each 3 -week cycle. Thirty minutes prior to paclitaxel administration, the patients were treated with the following premedications: dexamethasone $(20 \mathrm{mg})$, diphenhydramine $(50 \mathrm{mg}$ and a histamine receptor 2 (H2) blocker. The patients in the weekly arm were permittted to have their premedications altered to dexamethasone $(8 \mathrm{mg})$, diphenhydramine $(50 \mathrm{mg})$ and an $\mathrm{H} 2$ blocker. The glomerular filtration rate was substituted by the calculated value using the Cockroft equation. The treatment was continued up to a maximum of 6 cycles or until disease progression in both arms.

\section{Results}

Patient characteristics. A total of 402 patients with NSCLC were treated in our hospital between January 1999 and December 2010. Of the 218 patients with advanced-stage disease, 167 met the eligibility criteria. The characteristics of these 167 patients are summarized in Table I. All patients were Asian (Japanese, Korean or Chinese), the median patient age was 65 years (range, 31-79 years) and patients comprised 42 females and 125 males. The numbers of patients with adenocarcinoma, squamous cell carcinoma, large cell carcinoma and other types of carcinoma were 118, 44, 4 and 1, respectively. The weekly and standard arm regimens were used as front-line chemotherapies in 73 and 94 patients, respectively. In 108 patients, $\geq 1$ regimen of chemotherapy, including EGFR-TKI, was administered following the frontline chemotherapy. A history of EGFR-TKI treatment was reported in 66 patients, whereas the remaining 101 patients had not received EGFR-TKI treatment. There were no significant differences in age, gender, PS, clinical stage and smoking history between the 2 groups.

Treatment-related adverse effects. The treatment-related grade 2 or worse adverse effects observed in this study are summarized in Table II. Grade 3 or 4 neutropenia was observed in 37.0 and $70.2 \%$ of the patients in the weekly and standard arms, respectively, indicating that severe (grade 3 or 4) neutropenia was significantly more frequent in the standard $\operatorname{arm}(\mathrm{P}<0.0001)$. Grade 3 or 4 thrombocytopenia was observed in 11.0 and $12.8 \%$ of the patients in the weekly and standard arms, respectively $(\mathrm{P}=0.8127)$. Grade 3 or 4 peripheral neuropathy was observed in $0 \%$ and $8.5 \%$ of the patients in the weekly and standard arms, respectively $(\mathrm{P}=0.0188)$. Grade 4 neuropathy was not observed in the weekly arm, although it was observed in $3.2 \%$ of patients in the standard arm. The other hematological or non-hematological adverse effects observed were found to be moderate and manageable in both groups.

Tumor response. The tumor responses are listed in Table I. The ORRs of the weekly and standard arms were 37.0\% (95\% CI, 26.0-49.1) and 31.9\% (95\% CI, 22.7-42.3), respectively. The DCRs of the weekly and standard arms were $74.0 \%$ (95\% CI, 62.4-83.6) and $74.5 \%$ (95\% CI, 64.4-82.9), respectively. There were no significant differences in ORR and DCR between the 2 groups. 
Table I. Patient characteristics.

\begin{tabular}{|c|c|c|c|}
\hline Characteristics & Weekly arm (n=73) & Standard arm $(n=94)$ & P-value \\
\hline Age (years) & & & 0.2361 \\
\hline Median & 64 & 65.5 & \\
\hline Range & $31-78$ & $32-79$ & \\
\hline Gender & & & 0.3723 \\
\hline Female & 21 & 21 & \\
\hline Male & 52 & 73 & \\
\hline ECOG PS & & & 0.5402 \\
\hline $0-2$ & 61 & 85 & \\
\hline 3 or 4 & 12 & 9 & \\
\hline Smoking history & & & 0.7300 \\
\hline Never smoked & 19 & 27 & \\
\hline Past or current smoker & 54 & 67 & \\
\hline Histological diagnosis & & & $0.1581^{\mathrm{a}}$ \\
\hline Squamous cell carcinoma & 15 & 29 & \\
\hline Adenocarcinoma & 57 & 61 & \\
\hline Large cell carcinoma & 1 & 3 & \\
\hline Other & 0 & 1 & \\
\hline Initial clinical stage & & & 0.5890 \\
\hline IIIB & 19 & 21 & \\
\hline IV & 54 & 73 & \\
\hline EGFR-TKI treatment & & & 0.2667 \\
\hline Not used & 41 & 60 & \\
\hline Used & 32 & 34 & \\
\hline \multicolumn{4}{|l|}{ Objective response } \\
\hline $\mathrm{CR}$ & 1 & 2 & \\
\hline PR & 26 & 28 & \\
\hline $\mathrm{SD}$ & 27 & 40 & \\
\hline $\mathrm{PD}$ & 19 & 24 & \\
\hline ORR (\%) & 37.0 & 31.9 & 0.5143 \\
\hline $\operatorname{DCR}(\%)$ & 74.0 & 74.5 & 1.000 \\
\hline
\end{tabular}

ECOG, Eastern Cooperative Oncology Group; PS, performance status; EGFR-TKI, epidermal growth factor receptor tyrosine kinase inhibitor; $\mathrm{CR}$, complete remission; PR, partial remission; SD, stable disease; PD, progressive disease; ORR, objective response rate; DCR, disease control rate. a'Squamous vs. non-squamous cell carcinoma.

Table II. Adverse events ( $\geq$ grade 2 ) according to the treatment schedule.

\begin{tabular}{|c|c|c|c|c|c|c|c|c|c|}
\hline \multirow[b]{2}{*}{ Toxicity } & \multicolumn{4}{|c|}{ Weekly arm $(n=73)$} & \multicolumn{4}{|c|}{ Standard arm $(\mathrm{n}=94)$} & \multirow[b]{2}{*}{ P-value } \\
\hline & $\mathrm{G} 2$ & G3 & G4 & $\geq \mathrm{G} 3(\%)$ & G2 & G3 & G4 & $\geq \mathrm{G} 3(\%)$ & \\
\hline \multicolumn{10}{|l|}{ Hematological } \\
\hline Neutropenia & 15 & 19 & 8 & 37.0 & 6 & 25 & 41 & 70.2 & $<0.0001^{\mathrm{a}}$ \\
\hline Thrombocytopenia & 7 & 5 & 3 & 11.0 & 22 & 8 & 4 & 12.8 & 0.8127 \\
\hline Anemia & 34 & 9 & 6 & 20.5 & 38 & 17 & 9 & 27.7 & 0.4651 \\
\hline \multicolumn{10}{|l|}{ Nonhematological } \\
\hline Neuropathy & 0 & 0 & 0 & 0.0 & 9 & 5 & 3 & 8.5 & $0.0188^{a}$ \\
\hline Transaminase & 7 & 1 & 0 & 1.4 & 5 & 4 & 0 & 4.3 & 0.3875 \\
\hline Total bilirubin & 0 & 0 & 0 & 0.0 & 5 & 2 & 1 & 3.2 & 0.2574 \\
\hline Serum creatinine & 3 & 0 & 0 & 0.0 & 5 & 0 & 0 & 0.0 & n.d. \\
\hline
\end{tabular}

${ }^{\mathrm{a}} \mathrm{P}<0.05$. G, grade; n.d., not done. 
Table III. Univariate analysis of OS.

\begin{tabular}{lcc}
\hline Variable & MST (months) & P-value \\
\hline Weekly arm vs. standard arm & 11.8 vs. 11.6 & $0.3779^{\mathrm{b}}$ \\
Female vs. male & 17.1 vs. 10.4 & $0.0051^{\mathrm{a}}$ \\
Never smoked vs. smoker & 16.2 vs. 10.0 & $0.0043^{\mathrm{a}}$ \\
Age $<70$ vs. $\geq 70$ years & 11.6 vs. 11.8 & $0.6135^{\mathrm{b}}$ \\
PS $0 / 1 / 2$ vs. $3 / 4$ & 12.5 vs. 3.2 & $0.0062^{\mathrm{a}}$ \\
Non-sq vs. sq & 12.6 vs. 9.7 & $0.0280^{\mathrm{a}}$ \\
TKI used vs. never used & 16.5 vs. 9.7 & $0.0007^{\mathrm{a}}$ \\
Stage IIIB vs. IV & 14.6 vs. 10.7 & $0.0248^{\mathrm{a}}$ \\
Neutropenia (G3 or G4 vs. G1 or G2 vs. G0) & 13.3 vs. 10.7 vs. 9.7 & $0.0243^{\mathrm{a}}$ \\
Anemia G3 or G4 vs. G1 or G2 vs. G0) & 4.7 vs. 12.8 vs. 23.0 & $0.0002^{\mathrm{a}}$ \\
Thrombocytopenia (G3 or G4 vs. G1 or G2 vs. G0) & 5.2 vs. 12.6 vs. 10.6 & $0.3718^{\mathrm{b}}$
\end{tabular}

${ }^{\mathrm{a}} \mathrm{P}<0.05$. ${ }^{\mathrm{b}}$ not significant. OS, overall survival; MST, median survival time; PS, performance status; Sq, squamous cell carcinoma; TKI, tyrosine kinase inhibitor; $\mathrm{G}$, grade.

A os

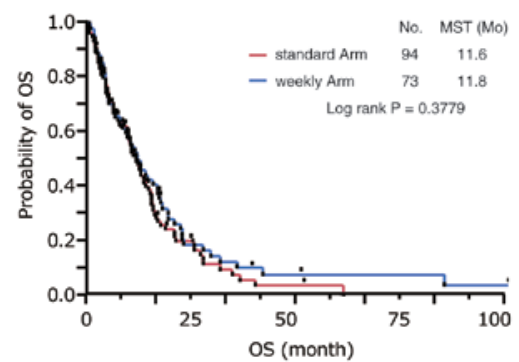

B PFS

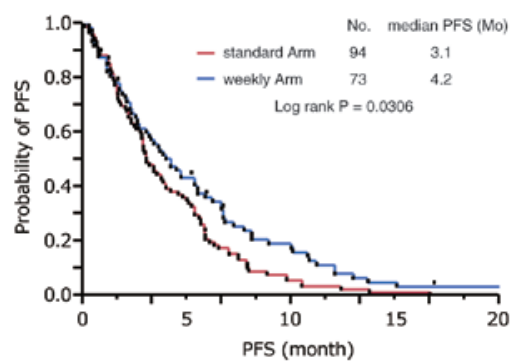

C OS per Neutropenia

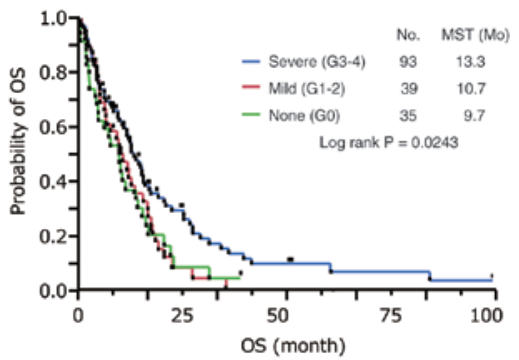

Figure 1. Kaplan-Meier curves of patients treated with carboplatin in combination with paclitaxel. (A) The Kaplan-Meier curves for overall survival (OS). The median survival times (MSTs) for the weekly and standard arms were 11.8 and 11.6 months, respectively (log-rank; P=0.3779). (B) The Kaplan-Meier curves for progression-free survival (PFS). The median PFS times for the weekly and standard arms were 4.2 and 3.1 months, respectively (log-rank; P=0.0306). (C) The Kaplan-Meier curves for OS according to the worst grade of treatment-related neutropenia. The MSTs for severe (grade 3 or 4 ), mild (grade 1 or 2 ) and absent (grade 0) neutropenia were 13.3, 10.7 and 9.7 months, respectively (log-rank; P=0.0243).

Survival data. We conducted a series of survival analyses on September 1, 2012. At that time, 136 patients had died, 28 patients had been lost to follow-up and 3 patients were alive. Consequently, the censoring rate was estimated at $18.6 \%$. The MSTs were 11.8 months (95\% CI, 8.4-16.8) and 11.6 months (95\% CI, 9.5-14.6) for the patients in the weekly and standard arms, respectively (Fig. 1A). The 1-year survival rates were 49.2\% (95\% CI, 37.1-61.3) and 48.8\% (95\% CI, 38.3-59.2) for the patients in the weekly and standard arms, respectively. The median PFS times were 4.2 months (95\% CI, 2.7-5.5) and 3.1 months (95\% CI, 2.8-4.0) for the patients in the weekly and standard arms, respectively (Fig. 1B). In the univariate analyses, the PFS was significantly longer in the weekly arm group $(\mathrm{P}=0.0306)$. The hazard ratio (HR) for the PFS of patients in the weekly arm versus the standard arm was $0.576(\mathrm{P}=0.0024)$.

Univariate analyses for OS. In the univariate analyses, the OS was significantly longer in the patients treated with EGFRTKIs $(\mathrm{P}=0.0007)$, females $(\mathrm{P}=0.0051)$, individuals who had never smoked $(\mathrm{P}=0.0043)$, those with $\mathrm{PS} 0 / 1 / 2(\mathrm{P}=0.0062)$, those with nonsquamous cell carcinoma $(\mathrm{P}=0.0280)$ and those with stage IIIB disease $(\mathrm{P}=0.0248)$, compared with the respective counterparts. However, the front-line chemotherapy schedules $(\mathrm{P}=0.3779)$ and patient age $(\mathrm{P}=0.6135)$ were not statistically significant prognostic factors for OS (Table III). We also analyzed the contribution of the worst grade of treatment-related hematological toxicity to OS. The correlation between OS and thrombocytopenia was not statistically significant $(\mathrm{P}=0.3718)$. The OS was significantly shorter in patients with severe anemia (grade 3 or $4 ; \mathrm{P}=0.0002$ ). On the contrary, the results of the alternative analyses clearly indicated that OS was significantly longer in patients with severe (grade 3 or 4 ) neutropenia ( $\mathrm{P}=0.0243$; Fig. 1C).

We had demonstrated that severe neutropenia more frequently occurred with the standard schedule (70.2 vs. $37.0 \%$ ), which indicated a significant survival impact, than in the weekly schedule; however, the survival curves of the 2 schedules almost overlapped (Fig. 1A). Subsequently, we conducted an alternative subanalysis for OS according to the worst grade of neutropenia (Fig. 2). In the subpopulations of mild (grade 1 or 2) and no (grade 0) neutropenia, comparable survival was demonstrated for the 2 schedules (Fig. 2A and B). 
Table IV. Multivariate analysis of OS.

\begin{tabular}{llll}
\hline Covariate & HR & $95 \%$ CI & P-value \\
\hline Weekly arm vs. standard arm & 0.634 & $0.422-0.948$ & $0.0262^{\mathrm{a}}$ \\
Female vs. male & 0.528 & $0.289-0.947$ & $0.0320^{\mathrm{a}}$ \\
Never smoked vs. smoker & 0.727 & $0.415-1.255$ & $0.2557^{\mathrm{b}}$ \\
Age $<70$ years vs. $\geq 70$ years & 1.078 & $0.714-1.647$ & $0.0002^{\mathrm{a}}$ \\
PS $0 / 1 / 2$ vs. $3 / 4$ & 0.296 & $0.167-0.550$ & $0.8501^{\mathrm{b}}$ \\
Non-sq vs. sq & 0.957 & $0.610-1.526$ & $0.0007^{\mathrm{a}}$ \\
TKI used vs. never used & 0.495 & $0.327-0.744$ & $0.0011^{\mathrm{a}}$ \\
Stage IIIB vs. IV & 0.485 & $0.301-0.756$ & $0.0007^{\mathrm{a}}$ \\
Neutropenia & & & $0.5392^{\mathrm{b}}$ \\
(G3 or G4 vs. G0) & 0.372 & $0.215-0.654$ & $0.0013^{\mathrm{a}}$ \\
(G1 or G2 vs. G0) & 0.825 & $0.448-1.527$ & $<0.0001^{\mathrm{a}}$ \\
(G3 or G4 vs. G1 or G2) & 0.450 & $0.281-0.728$ & $0.0056^{\mathrm{a}}$ \\
Anemia & & & $0.0001^{\mathrm{a}}$ \\
(G3 or G4 vs. G0) & 9.527 & $3.415-29.301$ & $1.422-9.775$ \\
(G1 or G2 vs. G0) & 3.514 & $1.641-4.404$ & $0.6290^{\mathrm{b}}$ \\
(G3 or G4 vs. G1 or G2) & 2.711 & & $0.2407^{\mathrm{b}}$ \\
Thrombocytopenia & & $0.524-1.709$ & $0.7538^{\mathrm{b}}$ \\
(G3 or G4 vs. G0) & 0.870 & $0.785-1.177$ & \\
(G1 or G2 vs. G0) & 1.108 & & \\
(G3 or G4 vs. G1 or G2) & &
\end{tabular}

${ }^{\mathrm{a}} \mathrm{P}<0.05 .{ }^{\mathrm{b}}$ not significant. OS, overall survival; HR, hazard ratio; CI, confidence interval; PS, performance status; Sq, squamous cell carcinoma; TKI, tyrosine kinase inhibitor; G, grade, ns, not significant.

A G0

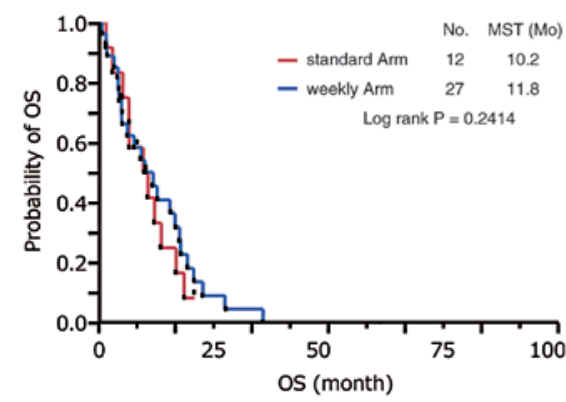

B G1orG2

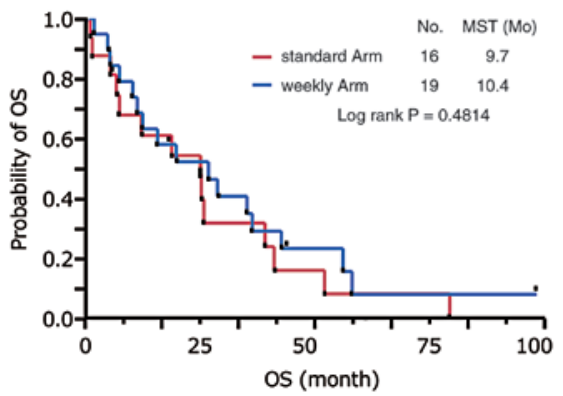

C $\quad$ G3 or G4

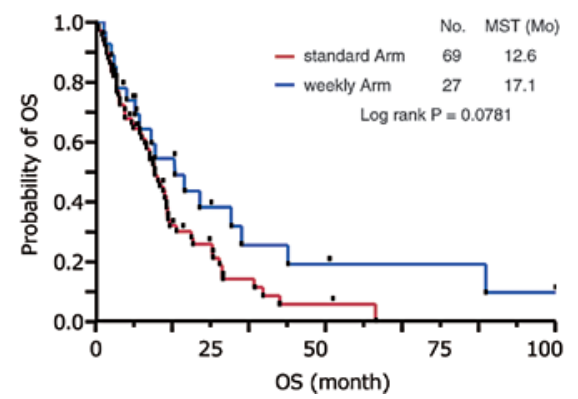

Figure 2. Kaplan-Meier curves of the patients according to the worst grade of treatment-related neutropenia. (A) The Kaplan-Meier curves for overall survival (OS) for the subgroup with grade 0 neutropenia. The median survival times (MSTs) for the weekly and standard arms were 11.8 and 10.2 months, respectively (log-rank; $\mathrm{P}=0.2414$ ). (B) The Kaplan-Meier curves for OS for the subgroup with grade 1 or 2 neutropenia. The MSTs for the weekly and standard arms were 10.4 and 9.7 months, respectively (log-rank; $\mathrm{P}=0.4814$ ). (C) The Kaplan-Meier curves for OS for the subgroup with grade 3 or 4 neutropenia. The MSTs for the weekly and standard arms were 17.1 and 12.6 months, respectively (log-rank; $\mathrm{P}=0.0781$ ).

However, in the subpopulation of severe (grade 3 or 4) neutropenia, the patients in the weekly schedule arm tended to have better survival that those in the standard schedule arm $(\mathrm{P}=0.0781$; Fig. 2C). To evaluate the independent survival impact of the covariates, we subsequently conducted a multivariate analysis that included the severity of hematological toxicities as covariates.

Multivariate analysis for OS. Unexpectedly, the multivariate analysis revealed that the weekly schedule was an independent favorable prognostic factor for $\mathrm{OS}(\mathrm{HR}=0.634 ; \mathrm{P}=0.0262)$, whereas the results of the univariate analysis failed to indicate a significant difference. In addition, PS $0 / 1 / 2(P=0.0002)$, stage IIIB disease $(\mathrm{P}=0.0011)$, a history of EGFR-TKI treatment $(\mathrm{P}=0.0007)$, female gender $(\mathrm{P}=0.0320)$, grade 3 or 4 neutropenia $(\mathrm{P}=0.0002)$ and grade 0 anemia $(\mathrm{P}<0.0001)$ were also independent favorable prognostic factors (Table IV). However, no significant difference in OS was observed between patients with grade 1 or 2 neutropenia and those with grade 0 neutropenia $(\mathrm{P}=0.5392)$. 


\section{Discussion}

The weekly schedule of paclitaxel and carboplatin combination treatment was investigated with 2 aims: one was to reduce the incidence of treatment-related toxicities, and the other was to assess the alternative antitumor effect based on the metronomic theory (16). To date, several weekly treatment schedules have been investigated (4-6). We applied the weekly schedule scheme (weekly arm) demonstrated by Hirabayashi et al (7). The efficacy and feasibility of this regimen were confirmed in an alternative phase II study described by Komuta et al (17). A previous randomized study described by Sakakibara et al compared these 2 schedule plans in an elderly patient ( $\geq 70$ years of age) setting and observed that in addition to its comparable survival benefit, the weekly schedule was less toxic than the standard schedule (18).

However, the close correlation between chemotherapy-related hematological toxicity and survival benefit has been discussed extensively for various malignant tumors (8-11). Kishida et al demonstrated that treatmentrelated neutropenia was a favorable predictive factor for the OS of patients with advanced NSCLC (JMTOG LC00-03) (9). Two additional studies have supported these findings $(10,11)$. We also demonstrated that grade 3 or 4 neutropenia was an independent favorable prognostic factor for OS in this study. However, the results of our univariate analyses clearly demonstrated that the OS times were similar between the 2 treatment schedules, whereas the incidence of grade 3 or 4 neutropenia was significantly lower in the weekly schedule arm than in the standard schedule arm. We hypothesized that an alternative survival benefit arising from the weekly schedule may overcome the disadvantage of the decreased incidence of neutropenia in that group. One possible explanation for this finding is that the metronomic activity of the weekly schedule may result in an alternative antitumor effect, leading to an additional survival benefit. Low-dose metronomic (LDM) chemotherapy consists of the administration of a relatively low dose of a cytotoxic drug without a long interval $(19,20)$. A number of experimental studies have demonstrated that LDM chemotherapy exerts an alternative antitumor effect different from the direct cytotoxic effect of the drug $(16,21)$. Notably, the result of our multivariate analysis revealed that the weekly schedule was an independent favorable prognostic factor for OS. Based on our rationale, optimization of the dose and schedule ought to achieve a superior survival benefit for the weekly schedule. Katsumata et al clearly demonstrated that a myelosuppressive 'dose-dense' weekly schedule has a superior survival benefit for ovarian cancer compared with the standard tri-weekly schedule (22). These findings strongly support our hypothesis. Therefore, the next question is whether escalation of the relative dose density leads to superior survival benefits. This will not be consistently true because the metronomic power is not augmented in a dosedependent manner. The maximum metronomic power of a drug is defined by the optimal biological dose (OBD) rather than the maximum tolerated dose (23). There is no reliable surrogate marker to determine OBD.

Despite the retrospective nature and small scale of the present study, our results clearly demonstrated that the weekly schedule of carboplatin and paclitaxel was less toxic than and potentially superior to the standard tri-weekly schedule. However, further modification of the dose and schedule is warranted.

\section{References}

1. D'Addario G, Pintilie M, Leighl NB, et al: Platinum-based versus non-platinum-based chemotherapy in advanced non-small-cell lung cancer: a meta-analysis of the published literature. J Clin Oncol 23: 2926-2936, 2005.

2. Ohe Y, Ohashi Y, Kubota K, et al: Randomized phase III study of cisplatin plus irinotecan versus carboplatin plus paclitaxel, cisplatin plus gemcitabine, and cisplatin plus vinorelbine for advanced non-small-cell lung cancer: Four-Arm Cooperative Study in Japan. Ann Oncol 18: 317-323, 2007.

3. Schiller JH, Harrington D, Belani CP, et al: Comparison of four chemotherapy regimens for advanced non-small-cell lung cancer. N Engl J Med 346: 92-98, 2002.

4. Belani CP, Ramalingam S, Perry MC, et al: Randomized, phase III study of weekly paclitaxel in combination with carboplatin versus standard every-3-weeks administration of carboplatin and paclitaxel for patients with previously untreated advanced non-small-cell lung cancer. J Clin Oncol 26: 468-473, 2008.

5. Socinski MA, Ivanova A, Bakri K, et al: A randomized phase II trial comparing every 3 -weeks carboplatin/paclitaxel with every 3 -weeks carboplatin and weekly paclitaxel in advanced non-small cell lung cancer. Ann Oncol 17: 104-109, 2006.

6. Quoix E, Zalcman G, Oster JP, et al: Carboplatin and weekly paclitaxel doublet chemotherapy compared with monotherapy in elderly patients with advanced non-small-cell lung cancer: IFCT-0501 randomised, phase 3 trial. Lancet 378: 1079-1088, 2011.

7. Hirabayashi M, Endoh K, Teramachi M, et al: Phase II study of carboplatin and weekly paclitaxel combination chemotherapy in advanced non-small cell lung cancer: a Kansai Clinical Oncology Group study. Lung Cancer 44: 355-362, 2004.

8. Shitara K, Matsuo K, Oze I, et al: Meta-analysis of neutropenia or leukopenia as a prognostic factor in patients with malignant disease undergoing chemotherapy. Cancer Chemother Pharmacol 68: 301-307, 2010.

9. Kishida Y, Kawahara M, Teramukai S, et al: Chemotherapyinduced neutropenia as a prognostic factor in advanced non-small-cell lung cancer: results from Japan Multinational Trial Organization LC00-03. Br J Cancer 101: 1537-1542, 2009.

10. Di Maio M, Gridelli C, Gallo C, et al: Chemotherapy-induced neutropenia and treatment efficacy in advanced non-small-cell lung cancer: a pooled analysis of three randomised trials. Lancet Oncol 6: 669-677, 2005.

11. Pallis AG, Agelaki S, Kakolyris S, et al: Chemotherapyinduced neutropenia as a prognostic factor in patients with advanced non-small cell lung cancer treated with front-line docetaxel-gemcitabine chemotherapy. Lung Cancer 62: 356-363, 2008.

12. Mountain CF: Revisions in the International System for Staging Lung Cancer. Chest 111: 1710-1717, 1997.

13. Sobin L and Wittekind CH (eds): TNM Classification of Malignant Tumours. 6th edition. Wiley-Liss, New York, NY, pp99-103, 2002.

14. National Cancer Institute (NCI): Common Terminology Criteria for Adverse Events (CTCAE) version 4.0. May 28, 2009. http:// evs.nci.nih.gov/ftp1/CTCAE/CTCAE_4.03_2010-06-14_ QuickReference_5x7.pdf. Accessed October 9, 2012.

15. Therasse P, Arbuck SG, Eisenhauer EA, et al: New guidelines to evaluate the response to treatment in solid tumors. European Organization for Research and Treatment of Cancer, National Cancer Institute of the United States, National Cancer Institute of Canada. J Natl Cancer Inst 92: 205-216, 2000.

16. Jiang H, Tao W, Zhang M, Pan S, Kanwar JR and Sun X: Low-dose metronomic paclitaxel chemotherapy suppresses breast tumors and metastases in mice. Cancer Invest 28: 74-84, 2010.

17. Komuta K, Osaki T, Mori M, et al: A phase II study directed by a clinical pathway for carboplatin and weekly paclitaxel in previously untreated patients with unresectable non-small cell lung cancer. Chemotherapy 56: 39-45, 2010.

18. Sakakibara T, Inoue A, Sugawara S, et al: Randomized phase II trial of weekly paclitaxel combined with carboplatin versus standard paclitaxel combined with carboplatin for elderly patients with advanced non-small-cell lung cancer. Ann Oncol 21: 795-799, 2010. 
19. Browder T, Butterfield CE, Kräling BM, et al: Antiangiogenic scheduling of chemotherapy improves efficacy against experimental drug-resistant cancer. Cancer Res 60: 1878-1886, 2000.

20. Drevs J, Fakler J, Eisele S, et al: Antiangiogenic potency of various chemotherapeutic drugs for metronomic chemotherapy. Anticancer Res 24: 1759-1763, 2004.

21. Kamat AA, Kim TJ, Landen CN Jr, Lu C, Han LY, Lin YG and Sood AK: Metronomic chemotherapy enhances the efficacy of antivascular therapy in ovarian cancer. Cancer Res 67: 281-288, 2007.
22. Katsumata N, Yasuda M, Takahashi F, et al: Dose-dense paclitaxel once a week in combination with carboplatin every 3 weeks for advanced ovarian cancer: a phase 3, open-label, randomised controlled trial. Lancet 371: 1331-1338, 2009.

23. Shaked Y, Emmenegger U, Man S, et al: Optimal biologic dose of metronomic chemotherapy regimens is associated with maximum antiangiogenic activity. Blood 106: 3058-3061, 2005. 